\title{
Implicit and Iterative Methods for the Boltzmann Equation
}

\author{
A.V. Bobylev* and J. Struckmeier \\ Department of Mathematics \\ University of Kaiserslautern \\ P.O. Box 3049 \\ 67653 Kaiserslautern \\ Germany
}

\begin{abstract}
The paper presents some approximation methods for the Boltzmann equation. In the first part fully implicit discretization techniques for the spatially homogeneous Boltzmann equation are investigated. The implicit equation is solved using an iteration process.

It is shown that the iteration converges to the correct solution for the moments of the distribution function as long as the mass conservation is strictly fulfilled.

For a simple model Boltzmann equation some unexpected features of the implicit scheme and the corresponding iteration process are clarified.

In the second part a new iteration algorithm is proposed which should be used for the stationary Boltzmann equation. The realization of the method is very similar to the standard splitting algorithms except some new stochastic elements.
\end{abstract}

${ }^{*}$ Permanent address: Keldysh Institute of Applied Mathematics, Academy of Sciences of Russia, Misskaya Sq. 4, 125047 Moscow, Russia 


\section{Introduction}

In the sequel we discuss some approximation methods for the numerical treatment of rarefied gas flows.

We consider the classical Boltzmann Equation (BE) for the distribution function $f(x, v, t)\left(x \in \mathbb{R}^{3}, v \in \mathbb{R}^{3}\right.$ and $t \in \mathbb{R}_{+}$denote respectively the space coordinate, velocity and time)

$$
\frac{\partial f}{\partial t}+v \cdot \frac{\partial f}{\partial x}=I(f, f)
$$

with the collision integral

$$
I(f, f)=\int_{\boldsymbol{R}^{3} \times S^{2}} g\left(|u|, \frac{u \cdot \omega}{|u|}\right)\left\{f\left(v^{\prime}\right) f\left(w^{\prime}\right)-f(v) f(w)\right\} \mathrm{d} w \mathrm{~d} \omega,
$$

and

$$
\begin{array}{lll}
u & =v-w \\
v^{\prime} & =\frac{1}{2}(v+w+|u| \omega) \\
w^{\prime} & =\frac{1}{2}(v+w-|u| \omega) \\
g(|u|, \cos \theta) & =|u| \sigma(|u|, \theta)
\end{array}
$$

$\omega \in S^{2}, 0 \leq \theta \leq \pi$

Here, $\sigma(|u|, \theta)$ denotes the differential cross-section at angle $\Theta \in[0, \pi]$ in the center of mass system.

The integrals

$$
\begin{aligned}
\sigma_{\text {tot }}(|u|) & =2 \pi \int_{0}^{\pi} \sigma(|u|, \theta) \sin \theta \mathrm{d} \theta \\
\sigma_{\text {tr }}(|u|) & =2 \pi \int_{0}^{\pi} \sigma(|u|, \theta) \sin \theta(1-\cos \theta) \mathrm{d} \theta
\end{aligned}
$$

are called respectively total and transport cross-sections. The function $g(|u|, \cos \theta)$ is called the collision kernel. It is important to distinguish the following two cases:

a) Maxwellian molecules with

$$
g(|u|, \cos \theta)=\tilde{g}(\cos \theta),
$$

i.e. the collision kernel is independent of $|u|$. 
b) Non-Maxwellian molecules, i.e. the general case when the collision kernel $g$ depends on $|u|$.

The special role of Maxwellian molecules for the BE is similar to the role of partial differential equations with constant coefficients in the general theory of PDE's. Therefore it is naturally to consider any problem (or method) first for the special case a) and then for the general case b). We use such an approach in the present paper.

Let us consider from a general point of view the problem of solving the Boltzmann equation (1) by numerical methods. On the basis of published materials (for a review see [3] and [11]) one may conclude the following:

A) The overwhelming majority of papers is based on the splitting algorithm for the $\mathrm{BE}$, i.e. the fractional step method is used and one solves the two equations

$$
\frac{\partial f}{\partial t}+v \cdot \frac{\partial f}{\partial x}=0 \quad \text { and } \quad \frac{\partial f}{\partial t}=I(f, f)
$$

for all time intervalls $[k \Delta,(k+1) \Delta], k \in I N_{0}$.

The first step (the so-called free motion) is simple enough. Concerning the second step (the spatially homogeneous BE) one may find in the literature a description of different explicit numerical schemes to solve it. A rigorous analysis of the splitting algorithm for the $\mathrm{BE}$ is given in [2] and [8].

B) Most applications in rarefied gas dynamics are given as stationary problems. Nevertheless, in general the non-stationary splitting algorithm as described in A) is used. Only very few numerical methods for the stationary $\mathrm{BE}$ are discussed in the literature [1].

Thus, one can easily think about at least two principal possibilities to improve the existing numerical schemes for the BE:

1) to use implicit schemes for solving the spatially homogeneous BE in the framework of fractional steps

and

2) to refuse the splitting algorithm and to use iterative schemes directly for the stationary BE. 
The main goal of the present paper is to describe some implicit and iterative methods and to discuss them in detail theoretically (advantages and possible realizations etc.) We do it first for the Maxwellian molecules and then generalize to the non-Maxwellian case.

Our considerations of the methods are oriented first of all to their numerical realization by the so-called Finite-Pointset-Method [9] - a particle method for the numerical treatment of the full Boltzmann equation. In this approach one is able to compute the collision integral $I(f, f)$ as well as the gain term $I_{+}(f, f)$ using a finite set of particles which approximate the distribution function $f$.

The concrete numerical realization of these new schemes by the FinitePointset-Method will be published elsewhere [12].

\section{Implicit Schemes for Maxwellian Molecules}

We consider the space uniform BE for Maxwellian molecules

$$
\frac{\partial f}{\partial t}=I(f, f)=\int_{\boldsymbol{R}^{3} \times S^{2}} g\left(\frac{u \cdot \omega}{|u|}\right)\left\{f\left(v^{\prime}\right) f\left(w^{\prime}\right)-f(v) f(w)\right\} \mathrm{d} w \mathrm{~d} \omega
$$

with notations (3). For simplicity we assume that

$$
\int_{S^{2}} g\left(\frac{u \cdot \omega}{|u|}\right) \mathrm{d} \omega=2 \pi \int_{-1}^{1} g(\eta) \mathrm{d} \eta=1
$$

and do not consider the case of true Mawellian molecules (particles interacting with the potential $U(r) \sim r^{-4}$ ). Then equation (7) and the corresponding initial condition may be written as

$$
\begin{array}{ll}
f_{t} & =\rho(F(f)-f) \\
\left.f\right|_{t=0} & =f_{0} \\
\rho & =\int_{\boldsymbol{R}^{3}} f(v) \mathrm{d} v
\end{array}
$$

and

$$
F(f)=\frac{1}{\rho} \int_{\boldsymbol{R}^{3} \times S^{2}} g\left(\frac{u \cdot \omega}{|u|}\right) f\left(v^{\prime}\right) f\left(w^{\prime}\right) \mathrm{d} w \mathrm{~d} \omega .
$$


Finally we assume that

$$
\left.\rho\right|_{t=0}=\int_{\boldsymbol{R}^{3}} f_{0}(v) \mathrm{d} v=1 .
$$

Then $\rho(t) \equiv 1$ for all $t>0$ because of mass conservation and equation (9) reads

$$
\begin{aligned}
f_{t} & =F(f)-f \\
\left.f\right|_{t=0} & =f_{0} \\
\int_{\mathbb{R}^{3}} f(v) \mathrm{d} v & =1
\end{aligned}
$$

The standard numerical scheme for this equation is based on the time discretization

$$
\left.\begin{array}{rl}
\frac{\hat{f}-f}{\Delta} & =F(f)-f \\
f & =f(t, \cdot) \\
\hat{f} & =f(t+\Delta, \cdot)
\end{array}\right\}
$$

so that the solution $\hat{f}$ is given by the explicit formula

$$
\hat{f}=\Delta F(f)+(1-\Delta) f
$$

with $(10)$ and $\rho=1$.

\section{Remark 1}

We do not distinguish here between concrete numerical realizations, in fact scheme (14) is used in nearly all stochastic as well as deterministic methods.

The scheme (14) has the important restriction $\Delta<1$ which is in some cases, as we shall see below, very inconvenient. One can easily construct models for the function $g(\eta)$ in $(10)$ such that the typical time scale of equation (12) is much less than the one in spite of conditions (8) and (11). The restriction on $\Delta$ is even more inconvenient for non-Maxwellian molecules.

Therefore it makes sense to consider a fully implicit discretization of (12) with arbitrary time step $\Delta$

$$
\frac{\hat{f}-f}{\Delta}=F(\hat{f})-\hat{f}
$$


and

$$
\hat{f}=\frac{\Delta}{1+\Delta} F(\hat{f})+\frac{1}{1+\Delta} f .
$$

In this case we have no restriction on $\Delta$ with respect to the positivity condition, but a new problem appears: how to solve equation (16).

A natural way is to consider the iteration process

$$
\hat{f}=\lim _{n \rightarrow \infty} f^{(n)},
$$

where

$$
\begin{aligned}
& f^{(0)}=f \\
& f^{(n+1)}=\frac{\Delta}{1+\Delta} F\left(f^{(n)}\right)+\frac{1}{1+\Delta} f
\end{aligned}
$$

but the convergence of the iterated sequence is not clear a priori. Therefore we consider this problem briefly in the next section.

\section{Convergence of the Iteration Process}

First of all we will show that the convergence of the iteration procedure defined by (17) is not trivial.

For example, suppose that we choose $f^{(0)}=0$ instead of the starting point $f^{(0)}=f$ as given in (17). Then we obtain

$$
\begin{aligned}
f^{(1)} & =\frac{1}{1+\Delta} f \\
f^{(2)} & =\frac{1}{(1+\Delta)^{2}} F(f)+\frac{1}{1+\Delta} f \\
& \vdots
\end{aligned}
$$

We notice that

$$
f^{(n+1)} \geq f^{(n)} \geq 0
$$

for all $n \in I N_{0}$, since

$$
f^{(n+1)}-f^{(n)}=\frac{\Delta}{1+\Delta}\left[F\left(f^{(n)}\right)-F\left(f^{(n-1)}\right)\right] \quad \forall n \in I N_{0}
$$

and

$$
f^{(1)} \geq f^{(0)}=0
$$


On the other hand, defining

$$
\begin{aligned}
& \rho^{(0)}=0 \\
& \rho^{(n)}=\int_{\boldsymbol{R}^{3}} f^{(n)}(v) \mathrm{d} v,
\end{aligned}
$$

we get

$$
\rho^{(n+1)}=\frac{1}{1+\Delta}\left[1+\Delta\left(\rho^{(n)}\right)^{2}\right] \leq 1 \quad \forall n \in I N_{0} .
$$

Hence, we have a monotonic sequence $0 \leq f^{(0)} \leq f^{(1)} \leq \ldots$ of integrable functions, for which the integrals

$$
\rho^{(n)}=\int_{\mathbb{R}^{3}} f^{(n)}(v) \mathrm{d} v \leq 1
$$

are uniformly bounded. Using the monotone convergence theorem [10] one can conclude that the sequence $\left\{f^{(n)}(v)\right\}_{n \in N}$ converges in $L_{1}\left(I^{3}\right)$ to the solution $\hat{f}$ of equation (16). To complete the proof we need to check the simple equality

$$
\lim _{n \rightarrow \infty} \rho^{(n)}=1
$$

with $\rho^{(n)}$ from (18). But in general the limit value for the sequence $\left\{f^{(n)}\right\}$ will be different from 1 !

The matter is that the iteration process (18) converges strictly to the minimal root of the quadratic equation

$$
\Delta \rho^{2}-(1+\Delta) \rho+1=0
$$

with roots

$$
\rho_{1}=1 \quad \text { and } \quad \rho_{2}=\frac{1}{\Delta},
$$

so that $\rho_{1}>\rho_{2}$ for $\Delta>1$. Thus, equation (16) has nonunique solutions and to obtain the "correct" one we need to fulfill strictly the condition

$$
\int_{\mathbb{R}^{3}} f^{(n)}(v) \mathrm{d} v=1 \quad \forall n \in I N_{0}
$$

This shows that the numerical realization of the iterative process (17) will be successful only if the numerical scheme to calculate $F(f)$ is strictly conservative with respect to mass conservation. 
We show now that the iteration process (17) does converge to the "correct" solution at least for the moments of the distribution function. For the proof we pass to the Fourier-representation of equation (16)

$$
\begin{aligned}
& \varphi(k)=\int_{\boldsymbol{R}^{3}} f(v) \mathrm{e}^{-i k v} \mathrm{~d} v \\
& \hat{\varphi}(k)=\int_{\boldsymbol{R}^{3}} \hat{f}(v) \mathrm{e}^{-i k v} \mathrm{~d} v .
\end{aligned}
$$

Then the implicit equation reads

$$
\hat{\varphi}=\frac{\Delta}{1+\Delta} \Phi(\hat{\varphi})+\frac{1}{1+\Delta} \varphi
$$

where

$$
\Phi(\hat{\varphi})=\int_{S^{2}} g\left(\frac{k \cdot \omega}{|k|}\right) \hat{\varphi}\left(\frac{k+|k| \omega}{2}\right) \hat{\varphi}\left(\frac{k-|k| \omega}{2}\right) \mathrm{d} \omega .
$$

Taking

$$
\begin{aligned}
& \varphi(k)=\sum_{n=0}^{\infty}|k|^{n} \varphi_{n}\left(\frac{k}{|k|}\right), \varphi_{0}=1 \\
& \hat{\varphi}(k)=\sum_{n=0}^{\infty}|k|^{n} \hat{\varphi}_{n}\left(\frac{k}{|k|}\right), \hat{\varphi}_{0}=1
\end{aligned}
$$

we obtain the set of equations

$$
\hat{\varphi}_{n}=\frac{\Delta}{1+\Delta} \sum_{l=0}^{n}\left\langle\hat{\varphi}_{l}, \hat{\varphi}_{n-l}\right\rangle+\frac{\varphi_{n}}{1+\Delta}
$$

with

$$
\left\langle\hat{\varphi}_{l}, \hat{\varphi}_{m}\right\rangle=\int_{S^{2}} g_{l m}\left(\frac{k \cdot \omega}{|k|}\right) \varphi_{l}\left(\frac{k+|k| \omega}{|k+| k|\omega|}\right) \varphi_{m}\left(\frac{k-|k| \omega}{|k-| k|\omega|}\right) \mathrm{d} \omega
$$

and

$$
g_{l m}(\cos \theta)=g(\cos \theta)\left(\cos \frac{\theta}{2}\right)^{l}\left(\sin \frac{\theta}{2}\right)^{m} .
$$

In a more compact form we get

$$
\hat{\varphi}_{n}=\frac{\Delta}{1+\Delta} L_{n} \hat{\varphi}_{n}+\frac{1}{1+\Delta} \varphi_{n}+\Psi_{n}\left(\hat{\varphi}_{1}, \ldots, \hat{\varphi}_{n-1}\right), \quad n>1
$$


with

$$
\begin{gathered}
\Psi_{n}\left(\hat{\varphi}_{1}, \ldots, \hat{\varphi}_{n-1}\right)=\sum_{l=1}^{n-1}\left\langle\hat{\varphi}_{l}, \hat{\varphi}_{n-l}\right\rangle \\
L_{n} \hat{\varphi}=\int_{S^{2}} g_{l m}\left[\cos ^{n} \frac{\theta}{2} \varphi\left(\frac{k+|k| \omega}{|k+| k|\omega|}\right)+\sin ^{n} \frac{\theta}{2} \varphi\left(\frac{k-|k| \omega}{|k-| k|\omega|}\right)\right] \mathrm{d} \omega
\end{gathered}
$$

and $\hat{\varphi}_{0}=\varphi_{0}$ respectively $\hat{\varphi}_{1}=\varphi_{1}$ - because of conservation of mass and momentum.

To prove the convergence of the iteration (17) for the equations (23) it is sufficient to notice that

a) the system (23) can be solved recursively, moreover the first equations $(n=0,1)$ are trivial,

b) the inequality

$$
\left\|L_{n} \varphi\right\| \leq\|\varphi\|=\sup _{\omega \in S^{2}}|\varphi(\omega)|
$$

follows from formula (24) and condition (8).

Finally we note that

$$
\varphi(k)=\sum_{n=0}^{\infty} \frac{|k|^{n}}{n !} \int_{\boldsymbol{R}^{3}} f(v)\left(-i \frac{k \cdot v}{|k|}\right)^{n} \mathrm{~d} v,
$$

i.e. the convergence of the iteration for $\varphi_{n}\left(\frac{k}{|k|}\right)$ is equivalent to the convergence of the moments of the distribution function.

\section{Remark 2}

Equation (16) has non-unique solutions; moreover the "correct" solution, satisfying the condition

$$
\rho=\int_{\boldsymbol{R}^{3}} f(v) \mathrm{d} v=1,
$$

is unstable (with respect to the convergence of the iteration) for all $\Delta>1$. However, we are able to avoid the instability if the condition (19) is fulfilled for all $n \in I N_{0}$. In particular, this is fulfilled automatically if we choose $f^{(0)}=f$.

In the next section we consider an example that shows some unexpected features of the implicit schemes. 


\section{A Model Boltzmann Equation}

Let us consider a special model for equation (7): we take

$$
g(\eta)=\frac{1}{2 \pi} \delta[\eta-(1-2 \varepsilon)], \quad 0 \leq \varepsilon \ll 1,
$$

which corresponds to a fixed scattering angle $\theta_{0}, \varepsilon=\sin ^{2} \frac{\theta_{0}}{2}$. We assume for simplicity that the distribution function is isotropic in velocity space, i.e. $f=f(|v|, t)$. Then we put

$$
\varphi\left(|k|^{2}, t\right)=\int_{\mathbb{R}^{3}} f(|v|, t) \mathrm{e}^{-i k \cdot v} \mathrm{~d} v
$$

and obtain - using $(22)$ - the following equation for $\varphi(z, t)\left(z=|k|^{2}\right)[5]$

$$
\left.\begin{array}{l}
\varphi_{t}=\varphi(\varepsilon z) \varphi[(1-\varepsilon) z]-\varphi(z) \\
\left.\varphi\right|_{t=0}=\varphi_{0}(z) .
\end{array}\right\}
$$

It is clear that both explicit (14) and implicit scheme (16) may be applied directly to this equation. It is moreover possible to compare the efficiency of both schemes for the moments of the distribution function $f(|v|, t)$.

We put

$$
\varphi(z, t)=\sum_{n=0}^{\infty}(-1)^{n} z^{n} \varphi_{n}(t)
$$

with

$$
\varphi_{n}(t)=\frac{1}{(2 n+1) !} \int_{\boldsymbol{R}^{3}} f(|v|, t)|v|^{2 n} \mathrm{~d} v \quad n \geq 0 .
$$

Substituting (28) into (27) we obtain quite simple equations for $\varphi_{n}(t)$ for all $n \in I N_{0}$

$$
\begin{aligned}
& \frac{\mathrm{d} \varphi_{0}}{\mathrm{~d} t}=\frac{\mathrm{d} \varphi_{1}}{\mathrm{~d} t}=0 \\
& \frac{\mathrm{d} \varphi_{n}}{\mathrm{~d} t}+\varphi_{n}=\sum_{l=0}^{n} \varepsilon^{l}(1-\varepsilon)^{n-l} \varphi_{l} \varphi_{n-l} .
\end{aligned}
$$

In our case $\varphi_{0}=\rho=1$, we also assume for simplicity

$$
\varphi_{1}(0)=\frac{1}{3 !} \int_{\mathbb{R}^{3}} f_{0}(|v|)|v|^{2} \mathrm{~d} v=1 .
$$


Then $\varphi_{1}(t)=1$, and the equation for $\varphi_{2}(t)$ reads

$$
\frac{\mathrm{d} \varphi_{2}}{\mathrm{~d} t}+2 \varepsilon(1-\varepsilon)\left[\varphi_{2}-\frac{1}{2}\right]=0,
$$

with exact solution

$$
\left.\begin{array}{rl}
\varphi_{2}(t) & =\frac{1-\mathrm{e}^{-\lambda t}}{2}+\varphi_{2}(0) \mathrm{e}^{-\lambda t} \\
\lambda & =2 \varepsilon(1-\varepsilon) .
\end{array}\right\}
$$

The typical relaxation time $\tau=\lambda^{-1}$ is very large for any $\varepsilon \ll 1$. If we use an explicit scheme (14) with $\delta<1$ then we need to perform a large number $N=O\left(\varepsilon^{-1}\right)$ of time steps to calculate the solution $f(|v|, t)$ for a characteristic time increment $t=\tau$.

One may expect that the numerical work can be reduced significantly using the implicit scheme (16) with time step $1 \ll \Delta \ll \varepsilon^{-1}-$ the restriction $\Delta \ll \varepsilon^{-1}$ being used here to reach a sufficient accuracy for our approximate solution.

Unfortunately, the situation is not so simple and we will show this on the basis of the almost trivial linear equation (31).

The explicit scheme (14) with maximal time step $\Delta=1$ results in the following formula for $\hat{\varphi}_{2}$ :

$$
\begin{aligned}
& \hat{\varphi}_{2}=\left[\varepsilon^{2}+(1-\varepsilon)^{2}\right] \varphi_{2}+\varepsilon(1-\varepsilon) \\
& \hat{\varphi}_{2}=\varphi_{2}(t+1) \\
& \varphi_{2}=\varphi_{2}(t)
\end{aligned}
$$

The implicit scheme (16) with arbitrary time step $\Delta$ reads

$$
\left.\begin{array}{rl}
\tilde{\varphi}_{2} & =\frac{\Delta}{1+\Delta}\left\{\tilde{\varphi}_{2}-2 \varepsilon(1-\varepsilon)\left[\tilde{\varphi}_{2}-\frac{1}{2}\right]\right\}+\frac{1}{1+\Delta} \varphi_{2} \\
\tilde{\varphi}_{2} & =\varphi_{2}(t+\Delta) \\
\varphi_{2} & =\varphi_{2}(t) .
\end{array}\right\}
$$

We define

$$
\begin{aligned}
y(t) & =\frac{\varphi_{2}(t)-\frac{1}{2}}{\varphi_{2}(0)-\frac{1}{2}} \\
\lambda & =2 \varepsilon(1-\varepsilon) \\
\Delta & =m
\end{aligned}
$$


with $1 \ll m \ll \lambda^{-1}$ and rewrite the equations (32)-(34) for the function $y(t)$. Then the exact solution (32) reads

$$
y(t)=\exp (-\lambda t)
$$

the explicit scheme (33) reads

$$
\begin{aligned}
y(0) & =1 \\
y(t+1) & =[1-\lambda] y(t)
\end{aligned}
$$

and for the implicit scheme (34) we obtain

$$
\begin{aligned}
y(0) & =1 \\
y(t+m) & =\frac{m}{1+m}(1-\lambda) y(t+m)+\frac{1}{1+m} y(t) .
\end{aligned}
$$

We remember that equation (37) with exact solution

$$
y(t+m)=\frac{y(t)}{1+\lambda m}
$$

should be considered in the framework of the iterative scheme (17), i.e.

$$
y(t+m)=\lim _{n \rightarrow \infty} y_{n}
$$

and

$$
\left.\begin{array}{rl}
y_{0} & =y(t) \\
y_{n+1} & =\frac{m}{1+m}(1-\lambda) y_{n}+\frac{1}{1+m} y_{0}
\end{array}\right\}
$$

for all $n \in I N$.

One can easily obtain the exact formula for $y_{n}$ in the form

$$
y_{n}=\frac{y_{0}}{1+\lambda m}\left\{1+\lambda m\left[\frac{m(1-\lambda)}{1+m}\right]^{n}\right\} \quad \forall n \in I N_{0}
$$

As we expected, it gives for $n \rightarrow \infty$ the exact formula (38). However in numerical calculations we always work with a finite number $n \in I N$ of iterations. To calculate $y(t+m)$ by the explicit scheme (14),(36) we do $m$ time steps and obtain

$$
y(t+m)=y(t)(1-\lambda)^{m} \quad \forall m \in I N_{0}
$$


Using the implicit scheme makes sense if the number $n$ of iterations is less then $m$, i.e. $n=\theta m, \theta \leq 1$. Therefore we consider (40) as a result of calculating $y(t+m)$ by the implicit scheme and obtain

$$
y(t+m)=\frac{y(t)}{1+\lambda m}\left\{1+\lambda m\left[\frac{m(1-\lambda)}{1+m}\right]^{\theta m}\right\}
$$

Finally we obtain the approximate solution for $t=N m$ in the form

$$
y(N m)=(1-\lambda)^{N m}
$$

for the explicit scheme and

$$
y(N m)=(1+\lambda m)^{-N}\left\{1+\lambda m\left[\frac{m(1-\lambda)}{1+m}\right]^{\theta m}\right\}^{N}
$$

for the implicit scheme.

It is clear that we should now pass to the limit $\lambda \rightarrow 0, m \rightarrow \infty, N \rightarrow$ $\infty, \lambda m \rightarrow 0$ and $\lambda N m=\lambda t$ is finite.

Using the limits above yields

$$
y(t) \rightarrow \exp (-\lambda t)
$$

for the explicit scheme, but

$$
y(t) \rightarrow \exp \left[-\lambda t\left(1-\mathrm{e}^{-\theta}\right)\right]
$$

for the implicit scheme. Hence, the implicit scheme (16) in connection with the iteration process (17) for the Boltzmann equation with the model function $g(\eta)$ as in (25) will have no advantages for a numerical method. To converge to the exact solutions it is necessary to do much more iterations $\left(\theta=\frac{n}{m} \rightarrow \infty\right)$ compared with the number of time steps in the explicit scheme.

We have considered in detail this simple example to illustrate some "inconvenient" and non-obvious feature of the implicit scheme (15)-(17): if one wants to minimize the numerical effort it is possible to loose the correct solution!

In our case the reason for the difficulties is caused by the iterative method (17), not by the implicit scheme (16) itself. To avoid the difficulties one should modify the iterative method (17) and consider other methods to solve equation (16), we suppose to return to this problem in a next paper.

Finally we generalize the implicit scheme (16) and the iteration process (17) to the case of non-Maxwellian molecules in the next section. 


\section{Non-Maxwellian Molecules}

We consider the spatially homogeneous Boltzmann equation

$$
\frac{\partial f}{\partial t}=I(f, f)=I^{+}(f)-\nu(f) f
$$

where $I(f, f)$ is defined in (2) and

$$
\left.\begin{array}{rl}
\nu(f) & =\int_{\mathbb{R}^{3}} f(w) g(|u|) \mathrm{d} w \\
g(u) & =u \sigma_{\text {tot }}(u)
\end{array}\right\}
$$

Furthermore we assume that the collision frequency $\nu(f)$ is bounded, i.e.

$$
\nu(f) \leq \nu_{M}=\text { const },
$$

with $\nu_{M}$ depending on the constant first moments of the distribution function. It means roughly that we approximate the function $g(|u|, \eta)$ in (2) by the function

$$
\tilde{g}(|u|, \eta)= \begin{cases}g(|u|, \eta) & |u|<R \\ g(R, \eta) & |u| \geq R\end{cases}
$$

for sufficiently large $R$. This kind of cut-off is generally used in numerical methods.

Now we are able to transform equation (43) to

$$
\frac{1}{\nu_{M}} \frac{\partial f}{\partial t}+f=F(f)
$$

with

$$
F(f)=\frac{1}{\nu_{M}} I^{+}(f)+\left[1-\frac{\nu(f)}{\nu_{m}}\right] f .
$$

If we put $\tau=\nu_{M} t$ then equation (47) reads

$$
f_{\tau}=F(f)-f
$$

similarly to equation (12) for Maxwellian molecules. We may also assume without restriction that

$$
\int_{\mathbb{R}^{3}} f(v) \mathrm{d} v=1
$$


Then all steps from section 2 to obtain formulas (13)-(17) may be repeated. For both - the Maxwellian as well as the non-Maxwellian case - the function $F(f)$ satisfies the conditions

$$
\int_{\mathbb{R}^{3}} F(f)\left\{\begin{array}{c}
1 \\
v \\
|v|^{2}
\end{array}\right\} \mathrm{d} v=\int_{\boldsymbol{R}^{3}} f(v)\left\{\begin{array}{c}
1 \\
v \\
|v|^{2}
\end{array}\right\} \mathrm{d} v .
$$

\section{Remark 3}

In the Maxwellian case one has $\nu(f)=\nu_{M}=1$.

It is clear that implicit schemes are extremely convenient for the nonMaxwellian case since we have very strong restrictions on the time step $\Delta$ (especially $\Delta \nu_{M}$ has to be less than 1) in the explicit scheme.

Another example for the importance of implicit schemes is perhaps their applications to particles interacting with long range potential, even with cut-off potentials.

In this case the typical relaxation time is defined by $\sigma_{\operatorname{tr}}(|u|)$ and not $\sigma_{\text {tot }}(|u|)$ (see equation 4 ). The value of $\sigma_{\text {tot }}(|u|)$ depends on the cut-off parameter and may be very large. At the same time this large value (similar to $\nu_{M}$ in (47)) defines very restricted conditions for an explicit scheme. Thus, using implicit schemes for such cases (for example, Coulomb potential with Debye screening) one can get essential advantages. However it is necessary to be careful enough to avoid some difficulties similar to the one described in the previous section.

\section{Iterative Methods for the Stationary Boltzmann Equation}

The majority of applications in rarefied gas dynamics are given as stationary flow problems (for example the flow around a reentry vehicle at high altitudes).

On the other hand, the numerical treatment, like the DSMC method [4], is based on an instationary flow simulation. The time variable is used like an iteration parameter to reach the stationary state.

The main disadvantage in this approach is that the time discretization has to be small enough to ensure that the fractional steps used to solve the instationary problem yields a suffiently accurate approximation of the exact steady state (see [8]). 
A direct method will be to use an iteration process for the stationary Boltzmann equation. In the following we introduce a quite simple iteration which results in an exact solution of the stationary equation.

We again start with Maxwellian molecules and the stationary Boltzmann equation

$$
\frac{1}{\rho(x)} v \cdot \frac{\partial f}{\partial x}+f=F(f)
$$

with notations (9) and (10). We further assume that some general boundary conditions in the form

$$
\left.f^{+}\right|_{\Gamma_{i}}=R_{i} f^{-}
$$

at fixed surface $\Gamma_{i}, i=1, \ldots, N$ are given [7].

Let us first recall shortly the (slightly modified) standard splitting method. Here one uses fractional steps, i.e. one considers the two simplified equations

$$
\frac{\partial f}{\partial t}+\left[\frac{1}{\rho(x)} v \cdot \frac{\partial f}{\partial x}\right]=0
$$

and

$$
\frac{\partial f}{\partial t}=F(f)-f
$$

The linear equation (50) can be solved without any difficulties (if we suppose that $\rho(x)$ is given). Neglecting the boundary conditions (49) the solution of the initial value problem is

$$
f(x, v, t)=f(x-v \tau(x, v, t), v, 0),
$$

where $\tau(x, v, t)$ is defined implicitely by

$$
t=\int_{0}^{\tau} \rho(x-v s) \mathrm{d} s .
$$

\section{Remark 4}

a) To take into account the boundary conditions is a well-known procedure.

b) Equation (48) may be simplified in the one-dimensional case $x \in I R$ by introducing the new variable

$$
y=\int_{0}^{x} \rho\left(x^{\prime}\right) \mathrm{d} x^{\prime}+\text { const. }
$$


The solution of the spatially homogeneous equation (51) was already discussed in the preceeding sections.

The natural way to solve directly the stationary equation (48) will be to consider the iterative process

$$
\begin{gathered}
\frac{1}{\rho_{n}(x)} v \cdot \frac{\partial f_{n+1}}{\partial x}+f_{n+1}=F\left(f_{n}\right) \\
\left.f_{n+1}^{+}\right|_{\Gamma_{i}}=R_{i} f_{n+1}^{-} \quad \forall n \in I N_{0}
\end{gathered}
$$

with an appropiate choice of the initial approximation function $f_{0}(x, v)$. It should be mentioned that there were attempts to apply similar schemes to boundary value problems (see for example [14]), but later they were almost refused in favour of splitting methods.

The main problem using this kind of iteration is to invert numerically the operator $A$ defined by

$$
A=\left[\frac{1}{\rho(x)} v \cdot \frac{\partial}{\partial x}\right]+1,
$$

where the brackets should indicate that we have incorporated the boundary conditions (49).

This problem seems to be more complicated than the problem of solving equation (50). However we can "reduce" the first problem to the second one.

Defining

$$
\begin{aligned}
D & =\left[\frac{1}{\rho(x)} v \cdot \frac{\partial}{\partial x}\right] \\
A & =1+D
\end{aligned}
$$

one may use the well-known operator identity

$$
(1+D)^{-1}=\int_{0}^{\infty} \mathrm{e}^{-t} \mathrm{e}^{-t D} \mathrm{~d} t
$$

to solve equation (52).

Denoting the operator $D_{n}$ as

$$
D_{n}=\left[\frac{1}{\rho_{n}(x)} v \cdot \frac{\partial}{\partial x}\right]
$$


we may write the formal solution of (52) in the form

$$
f_{n+1}(x, v)=\left\langle\mathrm{e}^{-t D_{n}} F\left(f_{n}\right)\right\rangle,
$$

where the brackets denote a time-averaging over the Poisson distribution

$$
\langle\Psi(t)\rangle=\int_{0}^{\infty} \mathrm{e}^{-t} \Psi(t) \mathrm{d} t
$$

Now the term $F\left(f_{n}\right)$ corresponds to an explicit discretization of equation (51) with $\Delta=1$, i.e. using the explicit discretization of

$$
\frac{\partial f}{\partial t}=F(f)-f
$$

in the form

$$
\frac{\tilde{f}-f}{\Delta}=F(f)-f
$$

with initial condition $f_{n}$ and time step $\Delta=1$ yields

$$
\tilde{f}=F\left(f_{n}\right)
$$

On the other hand, the solution of equation (50) can be written as

$$
f(x, v, t)=\mathrm{e}^{-t D} f(x, v, 0)
$$

and the classical splitting method given by (50) and (51) with $\Delta=1$ may be written as

$$
f_{n+1}=\mathrm{e}^{-D_{n}} F\left(f_{n}\right)
$$

Comparing (56) and (58) one recognizes the similarity between the standard splitting method and the new stochastic iteration approach:

Instead of a fixed time step $\Delta=1$ for equation (50) - as in the standard splitting method - one should use a Poisson distributed timestep in connection with the explicit discretization and $\Delta=1$.

It is clear that for the standard splitting approach with $\Delta=1$, i.e. a quite "large" time step we will not converge to the true solution of the Boltzmann equation (48). But we do converge (at least formally), if we add some stochasticity, i.e. a Poisson distributed time step to solve equation $(56)$ ! 
The generalization to the non-Maxwellian case is self-evident: similarly to (47), we write down the stationary BE in the form

$$
\frac{1}{\nu_{M}(x)} v \cdot \frac{\partial f}{\partial x}+f=F(f)
$$

and repeat all previous steps.

The only difference is that we use the "maximal collision frequency" $\nu_{M}(x)$, instead of the density $\rho(x)$, therefore all our considerations remain valid for the general (non-Maxwellian) case.

\section{Conclusions}

The overwhelming majority of numerical methods for the Boltzmann equation is based on the splitting algorithm with an explicit discretization of the spatially homogeneous Boltzmann equation. In this paper we discuss some other approaches, namely fully implicit schemes for the spatially homogeneous Boltzmann equation and iterative schemes for the full stationary Boltzmann equation.

Possible advantages of implicit schemes are obvious, but the problem of solving the implicit equation on each time level is non-trivial. We construct an iterative scheme to solve it and prove the convergence of the iterations for the moments of the distribution functions in the case of Maxwellian molecules. The main attention is devoted to some important features of the iterative procedure.

Furthermore we investigate the special role of mass conservation: for non-conservative approximation of the Boltzmann equation the iterations converge to the "incorrect" solution (see section 3).

The example in section 4 shows that there exist some correlations between the time step and the number of iterations. If we try to minimize the numerical effort then we should be careful since it is possible to get a convergence to an "incorrect" solution. One may obtain serious improvements using implicit schemes, but it is necesssary to be careful enough in practical realizations. The implicit schemes are obviously generalized to the non-Maxwellian case. We should mention that the implicit schemes may be used to construct second order accurate methods in time [13].

In the last section we consider an iterative method for the stationary Boltzmann equation and suggest a "stochastic" realization of this method that is formally very similar to the standard splitting algorithm. An unexpected result is as follows: to get a convergence to the solution of the 
stationary Boltzmann equation in the new splitting scheme we do not need to reduce (as far as we can) the discrete time step. Instead of it we consider the time step for the free motion as a random variable with Poisson distribution. It is shown that the realization of this procedures is equivalent to an iterative scheme for the stationary Boltzmann equation.

This new splitting method is especially convenient if one uses it in combination with the Finite-Pointset-Method [9]. The numerical realization and some results of this approach to the stationary Boltzmann equation will be discussed in a next paper [6].

\section{Acknowledgement}

A.V. Bobylev is grateful to the DFG (German Research Foundation) for the financial support and to H. Neunzert for his kind hospitality in Kaiserslautern and helpful discussions. The support of the Basic Research Foundation of Russia (Grant 93-01-01689) is also acknowledged.

\section{References}

[1] H. Babovsky (1992), Time Averages of Simulation Schemes as Approximations to Stationary Kinetic Equations, Eur. J. Mech., B/Fluids, Vol. 11, No. 2, 199-212.

[2] H. Babobsky and R. Illner (1989), A Convergence Proof for Nanbu's Simulation Method for the Full Boltzmann Equation, SIAM J. Numer. Anal., Vol. 26, No. 1, pp. 45-65.

[3] A. Beylich (Ed.) (1991), Proceedings of the 17th Int. Symp. on Rarefied Gas Dynamics, Weinheim.

[4] G.A. Bird (1994), Molecular Gas Dynamics and Direct Simulation of Gas Flows, Clarendon Press, Oxford.

[5] A.V. Bobylev (1988), Theory of space uniform Boltzmann equation for Maxwell molecules, Sov. Sci. Rev. C, 7, 111-233.

[6] A.V. Bobylev and J. Struckmeier, Numerical Solution of the Heat Transfer Problem for the Staionary Boltzmann Equation by the Method of Stochastic Iterations, to be published. 
[7] C. Cercignani, R. Ilner and M. Pulvirenti (1994), The Mathematical Theory of Dilute Gases, Springer, New York.

[8] L. Desvilettes and S. Mischler (1994), About the splitting algorithm for the Boltzmann and BGK Equation, preprint, University of Orleans, URA 1803.

[9] H. Neunzert and J. Struckmeier (1992), The Finite-Pointset-Method for Hypersonic Flows in the Rarefied Gas Regime, J.J. Bertin, R. Glowinski and J. Periaux (Eds.), Hypersonics, Vol. III, Birkhäuser.

[10] M. Reed and B. Simon (1972), Methods of Modern Mathematical Physics I: Functional Analysis, Academic Press, New York.

[11] B.D. Shizgal and D.P. Weaver (Eds.) (1994), Rarefied Gas Dynamics: Theory and Simulations, Progress in Astronautics and Aeronautics, Volume 159.

[12] J. Struckmeier, Implicit Particle Methods for the Boltzmann Equation with Maxwellian Molecules, to be published.

[13] J. Struckmeier and K. Steiner, Second Order Scheme for the Spatially Homogeneous Boltzmann Equation with Maxwellian Molecules, to be published.

[14] F.G. Tcheremissine (1970), Numerical solution of the Boltzmann Equation for one-dimensional stationary flows, (in Russian), Zh. Vych. Mat. \& Mat. Fiz., Vol. 10, No. 3, pp. 15-25.

Jens Struckmeier

Department of Mathematics

University of Kaiserslautern

P.O. Box 3049

67653 Kaiserslautern

Germany

E-mail: struckm@mathematik.uni-kl.de 